\title{
Advanced Processing of Functional Magnetic Materials
}

\author{
S.K. MCCALL (i) ${ }^{1,3}$ and I.C. NLEBEDIM (1) ${ }^{2,4}$ \\ 1.-Lawrence Livermore National Laboratory, Livermore, CA 94551, USA. 2.-Ames \\ Laboratory, Ames, IA 50011, USA. 3.—e-mail: mccall10@llnl.gov. 4.—e-mail: nlebedim@ameslab.gov
}

Functional materials, specifically those which respond to external stimuli, such as magnetic and electrical fields, are increasingly important for many green energy technologies. They are being investigated from basic and applied sciences perspectives. Probably the best known of these materials are permanent magnets, which are used to supply a magnetic field and are widely applied in motors, generators, data storage, sensors, and actuators. Other examples of functional materials include soft magnets, magnetocalorics, and thermoelectrics.

For many of these materials, the key properties of interest are highly sensitive to crystallographic orientation and the details of the microstructure. Thus, processing conditions can be nearly as important as the sample chemistry. Advanced processing routes such as additive manufacturing may lead to improved performance of functional magnetic materials or enable scaled production of materials that were previously laboratory curiosities due to the low production efficiencies of traditional methods. They can also enable rapid prototyping and minimize waste of expensive elements.

This special topic contains a set of papers exploring some new approaches to the manufacture of functional materials and the challenges in producing these materials with the desired properties. They highlight important aspects of tailoring the functionality of these materials for desired applications and include contributions on rare earth element (REE)-free permanent magnets, exchanged coupled magnetic composites, additive manufacturing of soft magnetic materials, deconvolution of contributions from multiphase magnetocaloric composites, and cold spray deposition of thermoelectric materials.

S. K. McCall and I. C. Nlebedim are Advisors for the TMS Magnetic Materials Committee and Guest Editors for the special topic Advanced Processing and Additive Manufacturing of Functional Magnetic Materials.
Sarkar and Mallick provide a detailed review of $\mathrm{MnBi}$ in "Synthesizing the hard magnetic lowtemperature phase of $\mathrm{MnBi}$ alloy: Challenges and Prospects." $\mathrm{MnBi}$ is the strongest REE-free permanent magnet and provides an advantageous departure from the typical response of coercivity (a measure of resistance to demagnetization) with changes in temperature; i.e., coercivity increases with increasing temperature for $\mathrm{MnBi}$. Despite the simple stoichiometry, successfully producing $\mathrm{MnBi}$ is remarkably difficult in part due to the phase of interest only forming in the solid state at comparably modest temperatures, transforming from the desired strong ferromagnetic phase to an undesired antiferromagnetic allotrope when heated to near $360^{\circ} \mathrm{C}$. Thus, scalable processing remains a challenge for this potentially new class of REEfree magnets. The history, challenges, cost implications, and future opportunities for permanent magnets based on $\mathrm{MnBi}$ are described in this review article.

A separate approach to improving the performance of REE-free permanent magnets is discussed by Zhang et al. in "Microstructure and Performance of $\mathrm{Mn}-\mathrm{Ga} / \mathrm{Fe}-\mathrm{Cr}-\mathrm{Co}$ Magnetic Composites Fabricated by Mechanical Alloying." In this work, the authors investigate $\mathrm{Mn}-\mathrm{Ga} / \mathrm{Fe}-\mathrm{Cr}-\mathrm{Co}$ magnetic composites by mechanical alloying in an effort to produce an exchange spring magnet. The concept behind such magnets is to couple a highly coercive material with a magnetically softer material that has a higher remanence. When combined at suitable length scales, the resulting hybrid system has a higher coercivity than the soft magnet, a higher remnant magnetization than the hard phase, and an overall improved energy product, a key figure of merit for permanent magnets.

Soft magnets have low coercivities and high magnetic permeability for preference. They find use in directing magnetic fields and providing shielding for sensitive instrumentation. In "General Investigations on Manufacturing Quality of Permalloy via Selective Laser Melting for 3D Printing 
of Customized Magnetic Shields," Li et al. describe their work with selective laser melting (SLM) for 3D printing of soft magnetic shields, addressing approaches to alleviate grain boundaries and cellular walls which can act as pinning centers to increase magnetic coercivity due to decreased magnetic permeability. The authors reported the suitability of SLM for producing promising soft magnetic materials, including the relationship between laser processing conditions and the resultant functionalities.

The magnetocaloric effect describes reversible temperature changes when a suitable magnetic material is (de)magnetized and can be used for magnetic refrigeration. As a result, magnetocaloric cooling systems can replace the existing traditional vapor-compression refrigeration technology and eliminate their associated complications, such as the need for environmentally unfriendly and hazardous chemicals. In "Phase Deconvolution of Multiphasic Materials by the Universal Scaling of the Magnetocaloric Effect,' Diaz-Garcia et al. present a method to deconvolve mixed phase magnetocaloric materials which shows good agreement with experimental data. This enables them to determine the phase fractions of different magnetocaloric materials present in a composite which will improve predictive modeling of more complex magnetic refrigeration systems.
Finally, an article by Baker et al. describes "Cold Spray Deposition of Thermoelectric Materials." This is noteworthy because much of the understanding of cold spray presumes the sprayed particles plastically deform to adhere to surfaces, yet the brittle thermoelectric Bi2Te3 is shown to deposit on both ductile and brittle surfaces, enabling any desired thickness to be obtained. Thermoelectric materials enable conversion between thermal and electrical energy, and can be used for applications like energy harvesting. The team demonstrated both $n$ - and $p$ type thermoelectrics sprayed onto the surface of a pipe, making a simple thermoelectric generator with excellent thermal coupling to a complex surface.

This work was supported by the Critical Materials Institute, an Energy Innovation Hub funded by the U.S. Department of Energy, Office of Energy Efficiency and Renewable Energy, Advanced Manufacturing Office. Work prepared by LLNL under Contract DE-AC52-07NA27344 and by Ames under contract DE-AC02-07CH11358.

To read or download any of the papers in this special topic, follow the URL http://link.springer.c om/journal/11837/72/8/page/1 to the table of contents page for the August 2020 issue (vol. 72, no. 8).

Publisher's Note Springer Nature remains neutral with regard to jurisdictional claims in published maps and institutional affiliations. 